\title{
Azoospermies non obstructives ; facteurs prédictifs du prélèvement testiculaire et risques de la fécondation assistée
}

\author{
Yigal SOFFER \\ Centre d'Infertilité Masculine, CHU Assaf Harofé - Zérifin, Faculté de Médecine Sackler, \\ Université de Tel Aviv, Tel Aviv, Israël
}

\section{RESUME}

Le prélèvement chirurgical de spermatozoïdes a révolutionné le traitement des azoospermies et plus particulièrement des azoospermies non-obstructives. Grâce à la microinjection intracytoplasmique (ICSI) qui permet à des spermatozoïdes défectueux de féconder l'ovocyte, des hommes naturellement inféconds peuvent engendrer. Ces techniques appliquées sans investigation préalable sur l'animal, ont soulevé un énorme enthousiasme et se pratiquent à grande échelle. Pour augmenter l'efficacité de ces traitements, les cliniciens cherchent, sans trop de succès, à mettre en évidence des facteurs de réussite qui leur permettraient de mieux conseiller les couples avec azoospermie non-obstructive, tributaires de ces techniques et d'éviter des interventions testiculaires inutiles et traumatisantes. Les recherches sur l'animal, entreprises après coup, soulèvent des réserves sur l'innocuité de l'ICSI et le bien-fondé d'une utilisation de ces spermatozoïdes, souvent à haut risque génétique. Plus encore, des travaux cliniques soulignent la fréquence inhabituelle de problèmes divers chez des enfants d'ICSI mais aussi de toute technique d'assistance à la reproduction. Ces observations troublantes ne sont pourtant pas corroborées par des suivis cliniques bien menés d'enfants d'ICSI. Ce paradoxe soulève une polémique vive.

Mots clés : infécondité masculine, azoospermies non-obstructives, prélèvements testiculaires, ICSI, techniques d'assistance à la reproduction, facteurs de succès, risques génétiques

\section{INTRODUCTION}

Grâce à la microinjection intra-cytoplasmique (ICSI) qui permet à des spermatozoïdes défectueux de féconder l'ovocyte, des hommes naturellement inféconds peuvent engendrer. Cette technique de fécondation assistée, appliquée à l'homme sans aucun préalable d'investigation sur l'animal, a soulevé un énorme enthousiasme et se pratique à grande échelle. Le prélèvement chirurgical de spermatozoïdes a révolutionné le traitement des azoospermies et plus particulièrement des azoospermies non-obstructives. Pour augmenter l'efficacité de ces traitements, les cliniciens cherchent à mettre en évidence des facteurs de succès qui leur permettraient de mieux conseiller les couples présentant une azoospermie nonobstructive, tributaires de ces techniques et d'éviter autant que possible des interventions inutiles et traumatisantes.

Mais des recherches sur l'animal, entreprises après coup, soulèvent des réserves sérieuses sur l'innocuité de la fécondation assistée et le bien-fondé d'une utilisation aveugle de spermatozoïdes déficients de sujets inféconds souvent à hauts risques génétiques sous-jacents, surtout dans les azoospermies non-obstructives, les formes les plus graves de l'infécondité masculine. Plus encore, de nombreux travaux cliniques soulignent la fréquence inhabituelle de problèmes divers chez des enfants issus d'ICSI mais aussi de toute forme de techniques d'assistance à la reproduction. Ces observations troublantes ne sont pourtant pas corroborées par des suivis cliniques bien menés d'enfants nés d'ICSI. Ce paradoxe soulève une vive polémique.

\section{Correspondance :}

Pr. Yigal Soffer - 19 Rue Herzog, IL 53601 Guivataïm. Israël Tel (972)3.571.82.13 - Fax (972)3.731.97.59 - Email ysoffer@tau.ac.il 
Après un bref historique, la littérature récente sur les facteurs de succès probables des prélèvements testiculaires dans les azoospermies non obstructives sera passée en revue. Puis ce sera le tour de la littérature sur les travaux expérimentaux de l'ICSI chez l'animal, les risques potentiels qui en découlent chez l'homme, les risques génétiques propres aux sujets tributaires de l'ICSI et les études cliniques des enfants nés de l'ICSI et d'autres assistances à la reproduction. Ces études concernent :

1 Des publications rétrospectives rapportant des problèmes inquiétants découverts chez ces enfants. Ces publications sont plus ou moins bien contrôlées et ne distinguent pas toujours l'ICSI des autres techniques d'assistance à la reproduction.

2 Des suivis prospectifs et prolongés d'enfants d'ICSI pour toutes indications, mieux contrôlés et plus rassurants, qui s'efforcent de tenir compte du contexte parental et socio-économique.

\section{HISTORIQUE}

La première micro injection intra-cytoplasmique d'un spermatozoïde (ICSI) dans un ovocyte humain a été pratiquée par Lanzendorf et coll., en 1988 [55].

Une fécondation normale a été observée dans quelques ovocytes, mais aucune grossesse n'en a résulté. Avec Palermo et coll., en 1992 [72], qui pratiquent à Bruxelles une micromanipulation plus raffinée, la répétitivité et la fiabilité de l'ICSI augmentent considérablement et des taux élevés de fécondations et de grossesses sont dès lors obtenus. Les premièrs prélèvements de sperme testiculaires avec ICSI sont faits en 1994 dans cette ville, par Schoysman et coll., [90] qui obtiennent la première grossesse d'une azoospermie non-obstructive, et par Devroye et coll. [24]. Ils sont rapidement suivis, d'abord à Bruxelles $[25,94]$ et partout dans le monde, d'une série ininterrompue de succès avec des grossesses nombreuses.

Le prélèvement du sperme se fait généralement par ponctions ou biopsies [35] et peut se répéter sans trop d'inconvénients [36] ce qui ne semble pas le cas dans un modèle animal, murin [91]. Les ponctions, moins invasives, donnent moins de spermatozoïdes que les biopsies multiples [35], mais les chances d'obtenir une grossesse ne semblent pas très différentes [2]. Les risques de complications sont relativement faibles $[82,125]$ et rarement sérieuses. Le prélèvement peut aussi se faire par une technique micro-chirurgicale, la micro-dissection décrite par Schlegel [89] qui permettrait, dans les cas difficiles, de mieux cibler les rares foyers résiduels de spermatogénèse et donnerait davantage de grossesses [113]

Après micro-injection des ovocytes ponctionnés, la congélation des spermatozoïdes surnuméraires, pratiquée par tous, augmente l'efficacité du prélèvement en permettant des cycles d'ICSI répétés avec du sperme décongelé dont le pouvoir fécondant est semblable à celui du sperme frais [37].

\section{FACTEURS DE SUCCES DU PRELEVEMENT DE SPERME TESTICULAIRE}

La qualité des spermatozoïdes testiculaires et leur viabilité [4], ainsi que leur morphologie [129] sont liées à leur degré de maturation et celle-ci est évidemment meilleure dans les azoospermies obstructives que dans les azoospermies non-obstructives, caractérisées par des troubles graves de la spermatogenèse. Le pronostic de ces azoospermies est lié avant tout au diagnostic étiologique qui requiert un bilan physique, échographique, endocrinien et génétique. Les résultats de ce bilan, les possibilités thérapeutiques et les risques doivent être discutés avec le couple avant tout traitement. Dans certains cas, comme dans les hypogonadismes hypogonadotropes, un traitement hormonal est possible et doit être entrepris avant d'envisager le prélèvement chirurgical. Dans la plupart des azoospermies non-obstructives, lorsqu'on ne peut trouver aucun spermatozoïde mature dans l'éjaculat, la recherche invasive de spermatozoïdes testiculaires pour ICSI est la seule option. Mais ces prélèvements testiculaires donnent, pour la plupart des auteurs, de moins bons résultats en ICSI que les prélèvements épididymaires ou testiculaires pour les azoospermies obstructives, avec une tendance à des taux de fécondation, d'implantations et de grossesses plus faibles [1, 46, $60,74,105]$. Certains auteurs présentent des résultats plus nuancés et parfois contradictoires [26, 39, 75, 115,120] d'où il ressort essentiellement que la qualité du développement embryonnaire précoce n'est pas affectée $[75,120]$. Mais pour d'autres, les résultats seraient pratiquement similaires [37, 95, 121] à l'exception d'un taux d'avortement significativement plus élevé dans les azoospermies nonobstructives [121].

Dans les azoospermies non-obstructives, la réussite du prélèvement de sperme reste peu prévisible. Le taux de succès général tourne autour de $40 \%[37,46,69,124]$. Ni l'âge du conjoint, ni son taux de FSH sérique ni même le compte-rendu histologique d'une biopsie testiculaire ne permettent de prédire d'une manière fiable la découverte de spermatozoïdes matures dans les testicules $[37,40,69$, 110]. Une analyse multifactorielle [37] confrontant tous les facteurs putatifs de succès dans les azoospermies nonobstructives montre effectivement que ces facteurs masculins n'ont aucun effet significatif sur les chances d'obtenir une grossesse clinique et évolutive.

Pour certains $[5,19]$, l'inhibine sérique $B$, marqueur fonctionnel des cellules de Sertoli signalerait bien la présence de spermatozoïdes matures dans les testicules, mais non pour d'autres [119, 122]. Le diagnostic histologique testiculaire serait encore l'indicateur relatif le plus intéressant [37, $63,108,110,121]$ mais ne peut servir qu'après une première intervention testiculaire. La biopsie testiculaire peut aussi être utilisé pour l'analyse en fluorescence des cellules de Sertoli [61, 77] à l'aide de marqueurs de l'hormone anti-Müllerienne et de la cytokératine-18 qui permettent de mesurer leur degré de maturité [61] ou bien pour la mesure en hybridation in situ fluorescente (FISH) de l'écartement des paires de chromosomes dans les spermatocytes $[77,130]$. La présence de nombreuses cellules de Sertoli 
matures, ainsi que d'une bonne proximité des chromosomes appariés dans les spermatocytes, prédiraient une spermatogenèse satisfaisante [130] avec de bonnes chances de trouver des spermatozoïdes matures. Mais ces travaux rétrospectifs n'ont pas encore été validés.

D'autres éléments pronostiques ont été suggérés qui ont l'avantage de ne pas exiger de biopsie testiculaire comme l'examen des délétions dans le bras long du chromosome $Y[49,71,92,93,96,100,101,111]$. On connaît aujourd'hui trois zones de délétions, dont deux, AZFa, et AZFb, sont plus rares mais d'un pronostic grave car elles prédisent l'échec du prélèvement spermatique [16,97]. Mais ces délétions n'ont de valeur prédictive que si elles sont détectées ce qui n'arrive que dans une minorité de cas [97]. Les délétions de la zone AZFC sont plus fréquentes mais compatibles avec des oligozoospermies diverses et même avec une fécondité maintenue [97]. Quant à l'analyse cytométrique de la ploïdie des cellules germinales dans l'éjaculat, elle donne un aperçu [56] de l'état de la spermatogenèse dans les testicules sans faire de biopsie. La méthode est facilement applicable mais non encore validée. L'analyse de la vascularisation des testicules [34] par échographie avec Doppler en couleur permettrait semble-t-il, à l'aide d'un système d'imagerie assistée sur ordinateur, de localiser les sites de spermatogénèse résiduelle dans les testicules afin de mieux cibler les ponctions et les biopsies à la recherche de spermatozoïdes matures.

Qu'en est-il de l'impact des facteurs féminins sur le succès de la fécondation assistée lorsqu'on a trouvé du sperme testiculaire pour ICSI ? L'âge de la femme et la réserve ovarienne, exprimée par le nombre des ovocytes ponctionnés ou injectés, ont une influence décisive sur le taux de succès de I'ICSI et cela indépendamment du type d'azoospermie [37, 95] et de l'histologie testiculaire [37] pour les azoospermies non-obstructives.

Pour l'âge de la femme, le seuil critique se trouverait entre 37 [95] et 38 ans [37] au delà duquel les chances de succès en ICSI tombent fortement. Pour le nombre minimum d'ovocytes ponctionnés, le seuil critique serait entre huit [95] et quatre [37]. II serait de trois pour les ovocytes injectés et matures [37].

\section{RISQUES GENETIQUES INHERENTS A LA MICRO INJECTION (Tableau 1)}

Sans la moindre donnée fiable concernant son innocuité, la technique de l'ICSI est devenue en très peu d'années une technique de routine de reproduction assistée. Aujourd'hui encore, douze ans après son introduction, l'expérience clinique dépasse de loin la recherche scientifique sur l'animal [86]. Plusieurs études expérimentales ont été lancées. En raison des différences entre espèces, il est fort douteux qu'elles puissent répondre aux multiples questions concernant l'innocuité de l'ICSI chez l'homme [112]. On ne peut aisément faire des extrapolations de l'animal à l'homme [86] concernant, le bloc de la polyspermie et le développement embryonnaire in vitro, entre autres, sans compter les pathologies humaines, souvent héréditaires, sous-tendant
Tableau 1 : Les risques de l'ICSI.

\section{- Risques inhérents à la procédure d'ICSI}

- Dégâts du fuseau méiotique ovocytaire.

- Trouble de l'activation ovocytaire, du déclenchement et de la décondensation de l'ADN spermatique.

- Défaut d'orientation des pronucléi mâle et femelle.

- Introduction de matériel étranger : PVP et ADN étrangère.

- Transmissions génétiques extra nucléaires et altérations épigénétiques

- Risques inhérents au spermatozoïde fécondant (défaut de sélection naturelle protectrice)

*Utilisation d'un spermatozoïde défectueux :

- Grosse anomalie morphologique.

- Cellules germinales immatures.

- Spermatozoïdes sénescents (apoptotiques).

- Risques génétiques paternels

*Syndromes héréditaires définis :

- Globozoospermies.

- Maladie de Kallmann et autres hypogonadismes similaires.

- Klinefelter et dysgénésies mixtes, Male XX.

- Prader Willy, Angelmann.

*Pathologies géniques et chromosomiques:

- Aberrations chromosomiques diverses.

- Translocations, Inversions, Aneuploidies diverses.

- Délétions Y.

- Répétitions CAG.

les infécondités masculines et plus particulièrement les azoospermie non-obstructives. Les modèles très coûteux d'ICSI sur primates $[44,85]$ semblent être les plus appropriés, mais ils ne concernent que des animaux bien portants et féconds et la controverse sur la nocivité de l'ICSI se poursuit.

Aussi, en dépit de la recherche sur l'animal, ce sont les grands suivis cliniques bien menés [112] d'enfants issus d'ICSI qui nous procurent aujourd'hui les données les plus fiables sur le développement physique et mental de cette progéniture [11, 12, 13, 14, 118].

\section{Pourquoi cette inquiétude?}

En FIV classique, les spermatozoïdes nageant autour de l'ovocyte entrent en compétition et la sélection du sperme fécondant se fait selon leurs caractéristiques de mobilité, de réaction acrosomique, de passage de la zone pellucide ou de fusion avec la membrane plasmatique. En ICSI, il n'en est plus de même. Dans une mise au point récente [87], Schatten souligne les aspects problématiques. 
1. C'est l'embryologiste qui choisit le spermatozoïde fécondant, selon des critères subjectifs fondés essentiellement sur l'aspect et la mobilité des spermatozoïdes au microscope. Aussi cette injection spermatique, aveugle d'une certaine façon, est assimilée par certains à un "viol de l'ovocyte" qui ne peut qu'éveiller des préoccupations graves et d'un ordre nouveau puisqu'elles ne concernent plus le sujet traité mais sa progéniture [87, 126].

2. Bien que la micro injection se fasse, selon les règles de l'art, loin du globule polaire, elle peut néanmoins endommager le second fuseau méiotique ovocytaire. Des études sur le hamster [98] et le singe Rhésus [44, 59] ont montré que même lorsque l'aiguille est en bonne position, à angle droit du premier globule polaire, le fuseau risque d'être lésé car la localisation du globule polaire ne témoigne pas en toute certitude de sa position actuelle. L'injection directe dans le cytoplasme peut-elle induire des fractures chromosomiques ? Les résultats publiés sont contradictoires $[8,30,81]$.

3. Même si l'aiguille de l'ICSI soigneusement introduite dans l'ovocyte, a pu éviter tout dégât du fuseau méiotique et permis une bonne restauration membranaire, on peut encore s'attendre à ce que l'activation, le déclenchement et la propagation de la réaction corticale se fassent autrement qu'en FIV. Ludwig et coll., 2003 [58], ont présenté une revue exhaustive sur l'activation et la fécondation ovocytaires en ICSI. La décondensation de l'ADN spermatique n'est pas non plus uniforme ; le premier cycle de réplication de l'ADN est retardé [45] et l'orientation des pronucléi mâle et femelle est perpendiculaire à celle qui est observée en FIV [86]. Des observations fascinantes sur l'axe corporel des mammifères montrent que les ovocytes murins et humains présentent une polarité intrinsèque $[38,45,132]$ et que, chez la souris, l'entrée du spermatozoïde [80] déterminerait un second axe. L'extrapolation, à l'homme, donnerait à penser que la région choisie pour l'introduction du spermatozoïde déterminerait le futur axe corporel gauche-droit [86].

4. L'introduction possible de matériel étranger dans le cytoplasme ovocytaire est également préoccupante. II s'agit du polyvinyle pyrrolidone (PVP) utilisé en ICSI pour ralentir le mouvement des spermatozoïdes, de microorganismes [18], vecteurs de contamination potentiels, ou de prions [54]. Un ADN étranger adhérent aux spermatozoïdes a pu être transmis en ICSI à des souris transgéniques [78], mais non en FIV chez des primates [21]

5. Les micromanipulations ovocytaires et la transmission génétique extra nucléaire sont devenues faisables en ICSI. Ce domaine de la biologie reproductive humaine n'est pas encore élucidé. Les mitochondries, d'origine exclusivement maternelles [23], peuvent être modifiées par transfert de mitochondries étrangères [17, 102].

6. Le fuseau mitotique polaire du zygote requiert la présence du centrosome spermatique. Pour de nombreux auteurs $[73,84,85,99,117]$ le dysfonctionnement de ce centrosome représenterait une forme d'infécondité masculine.

7. D'autres apports génétiques uni-parentaux proviennent des membranes endoplasmiques, de l'ARN spermatique [67] et d'altérations épigénétiques du patrimoine génétique nucléaire, causées par l'assemblage et la recombinaison d'éléments intracellulaires ou membranaires, selon des modes étrangers aux lois de la génétique. D'autres altérations épigénétiques seraient liées aux milieux de culture des gamètes [32].

8. Mais ce n'est pas tout : l'attrait de l'ICSI, qui permet à des hommes inféconds d'engendrer, peut masquer des risques génétiques graves, sous-jacents à la pathologie de cette infécondité paternelle.

a) Des aberrations chromosomiques nombreuses bien inventoriées dans la revue recente de Calogero et coll. 2003 [20] ont été découvertes dans les oligozoospermies et les azoospermies [9, 33, 62, 66, 76]. Ces anomalies autosomiques du caryotype se retrouvent, d'une manière inversement proportionnel au nombre des spermatozoïdes [131]. Si elles se retrouvent dans près de $3 \%$ dans les oligozoospermies modérées, elles atteignent près de $7 \%$ dans les oligozoospermies sévères et près du double dans les azoospermies non-obstructives. Bonduelle et coll., 1999 [13], signalent la présence d'anomalies chromosomiques dans près de $1 \%$ des enfants d'ICSI. Le risque d'aneuploïdie dans la progéniture d'ICSl reflète non seulement le taux plus élevé d'aneuploïdies dans ces pathologies mais aussi la survenue plus fréquente de cellules germinales aneuploïdes chez des sujets inféconds à caryotype normal [9]. Un dépistage rigoureux avec des méthodes plus performantes pourrait diminuer de manière substantielle ces risques.

b) Plus lourde de danger encore est l'utilisation en ICSI pour azoospermie non-obstructive, en l'absence de cellules matures, de spermatides immatures. Outre la difficulté de les distinguer des cellules diploïdes par leur simple apparence, leur expression génomique peut, pour Tesarik et Mendoza, [107], être incomplète avec tous les risques impliqués. Mais chez la souris, la descendance des ICSI avec spermatocytes secondaires est féconde [48] suggérant dans cette espèce une expression génomique complète à la seconde division méiotique ou capable de s'achever dans le cytoplasme ovocytaire. L'extrapolation de ces résultats à l'homme serait prématurée. La maturation in vitro est faisable [107]. Bien qu'aucun problème n'ait été jusqu'à présent rapporté chez des enfants nés de ce type de cellules immatures ou maturées in vitro elle pourrait ajouter des risques épigénétiques, La maturation de cellules germinales par transferts testiculaires [107] homologues ou hétérologues a été également expérimentée avec succès chez l'animal. Elle ne peut encore être envisagée chez l'homme. 
c) On ne trouve parfois dans l'éjaculat de certains hommes non azoospermiques que des spermatozoïdes sénescents ou morts dont la chromatine présente de multiples ruptures des chaînes d'ADN (apoptose). Des éjaculations répétées permettraient dans certains cas de trouver des spermatozoïdes plus viables et même mobiles [83] pouvant donner des grossesses en ICSI. Sinon, il serait préférable, chez eux, de ne pas utiliser ce sperme éjaculé en apoptose et de prélever des spermatozoïdes testiculaires de bonne vitalité [109].

d) Les délétions de gènes du bras long du chromosome $Y$ détectées chez certains hommes inféconds peuvent passer aux générations futures $[47,71,92$, 93, 96, 97, 111]. En outre des anomalies de répétitions CAG du récepteur d'androgène peuvent être liées à l'infécondité $[28,114]$. Ces répétitions peuvent non seulement causer une infécondité masculine mais aussi provoquer [22] l'Atrophie Musculaire de Kennedy (liée au sexe, spinale et bulbaire).

L'hérédité de l'infécondité masculine est encore loin d'être élucidèe. Mais il s'avère de plus en plus pour Meschede et coll. [64, 65], que des marques héréditaires, parfois détectables, sont impliquées. II semble bien exister des homologues autosomiques du chromosome $Y$ et d'autres gènes récessifs encore mal connus, impliqués directement dans la régulation de la spermatogenèse ou indirectement liés dans un système génétique modulateur plus complexe. II semblerait également que les candidats à l'ICSI présenteraient aussi une fréquence accrue de troubles de la sphère non reproductive potentiellement héréditaires. L'ICSI perpétuerait-elle ces formes d'infécondité masculine à une progéniture obligée, elle aussi, de recourir à cet artifice ?

Concernant la propagation des délétions du chromosome $Y$ dans la progéniture des hommes azoospermiques ou oligozoospermiques tributaires d'ICSI, il semblerait pour Kremer et coll. [51], que la fréquence de ces délétions finiraient, selon un modèle mathématique, par se stabiliser rapidement, même si l'ICSI devenait encore plus performant à l'avenir. II ne faut pas oublier que la perpétuation des formes héréditaires des infécondités masculines est aussi possible avec des thérapeutiques plus conventionnelles comme par exemple l'administration de gonadotropines dans la maladie de Kallmann, liée à la mutation du gène KALIG-1 [10]. Quant aux répétitions GAC, aucune augmentation de celles-ci n'a encore été observée chez les enfants d'ICSI [10].

Bien que les sujets tributaires d'ICSI présentent souvent de fortes tératozoospermies, de nombreuses études $[52,70]$ n'en ont déploré aucun résultat fâcheux ; ces travaux n'avaient cependant pas pris en ligne de compte la morphologie particulière du spermatozoïde micro-injecté. Dans les quelques travaux corrélant les résultats de l'ICSI avec cette morphologie spécifique, un taux réduit de fécondation a été rapporté $[60,106]$. Yanagimachi [128] a observé après micro-injection dans des ovocytes murins un taux plus élevé d'anomalies chromosomiques dans les spermatozoïdes humains présentant de fortes anomalies de la tête. Dans une étude, non encore publiée, présentèe à I'ESHRE de Madrid, 2003, Strassburger et coll. [103] ont trouvé des résultats similaires dans des spermatozoïdes humains sur des frottis analysés à l'aide d'un système d'imagerie couplée en microscopie à fort grossissement pour la morphologie stricte et en FISH pour les aberrations chromosomiques. II y aurait donc intérêt de perfectionner la sélection morphologique des spermatozoïdes à injecter. Il faut signaler à ce sujet le développement d'un appareillage particulier permettant de mieux observer en temps réel la morphologie des spermatozoïdes mobiles sous objectif Nomarsky de fort grossissement $(x 1000)$ avec agrandissement sur écran à $x 6300$. Dans une étude [6] non contrôlée, un nombre accru de grossesses aurajent été ainsi obtenues dans un groupe d'échecs répétés et inexpliqués d'ICSI avec des spermatozoïdes sélectionnés à l'aide de cet appareillage.

\section{RISQUES DES TECHNIQUES DE REPRODUC- TION ASSISTEE NON SPECIFIQUES DE L'ICSI ET DES AZOOSPERMIES NON-OBSTRUCTIVES} (Tableau 2)

"Ignorons-nous les dangers potentiels de la fécondation in vitro et des traitements associés" ? se demandent Winston et Hardy [126].

Les grossesses obtenues par technique d'assistance à la reproduction, de quelque type que ce soit, donnent des résultats légèrement moins bons que les grossesses conçues naturellement $[3,27,29,79,104]$. Ces risques concernent tous les couples tributaires des technique d'assistance à la reproduction et ne sont pas spécifiques de l'ICSI ou des azoospermies non-obstructives. Est-ce à cause des particularités spécifiques de la population rede-

Tableau 2 : Risques des techniques d'assistance à la reproduction (non spécifiques de l'ICSI et des azoospermies non-obstructives).

- Risques inhérents à la femme tributaire des techniques d'assistance à la reproduction

*Age plus avancés ; antécédents infectieux ; pathologies gynécologiques:

- Défaut de placentation, fausses couches, insuffisances placentaires, toxémies gravidiques, malformations congénitales, accouchements prématurés, faible poids de naissance.

- Risque des techniques d'assistance à la reproduction

* Hyper Stimulation ovarienne et transfert multiples d'embryons:

- Grossesses multiples, accouchements prématurés, faible poids de naissance.

* Méthylation des gamètes par les milieux de culture et modifications épigénétiques :

- Maladies rares et tumeurs malignes. 
vables des techniques d'assistance à la reproduction ? Ces femmes sont généralement plus âgées et ont plus de problèmes gynécologiques et infectieux ce qui ne peut qu'augmenter les risques obstétricaux et néonataux [12, $88,116,123,127]$. De plus, les stimulations ovariennes avec ovulations très nombreuses et les transferts multiples d'embryons sont responsables des grossesses gémellaires qui constituent les causes majeures des complications de grossesses et des pathologies néonatales. Dans un article très récent, Edwards \& Ludwig [32], font le point sur l'origine des anomalies majeures suite aux techniques d'assistance à la reproduction. Elles seraient dues à des cascades multifactorielles d'origines multiples, parentale, surtout paternelle, socio-économique, gynécologique et obstétricale. Ils n'excluent pas les risques épigénétiques pouvant survenir dans les ovocytes en milieux de culture y compris les phénomènes de déméthylation ainsi que certains effets des traitements associés des ART. Selon Gosden et coll. [42] ces modifications épigénétiques seraient à l'origine d'anomalies congénitales et de syndromes rares comme ceux d'Angelman et de Beckwith-Wiedemann rapportés avec une fréquence inhabituelle chez des enfants nés de techniques d'assistance à la reproduction. Des tumeurs malignes rares, comme le rétinoblastome, ont été rapportées $[7,68]$ chez des enfants de FIV ou d'ICSI. Est-ce aussi à cause de ces modifications épigénétiques ou des traitements associés de la FIV ?

Quoiqu'il en soit, la micro-injection ne semble y avoir qu'un rôle négligeable [32]. Dans un éditorial, Kovalevsky et coll. [50] recommandent de contrôler les études sur les dangers de l'ICSI et la FIV non seulement avec une population naturellement féconde mais aussi avec des couples inféconds traités à l'ancienne, sans ART.

Tournaye se demande [112], lui aussi, si l'on n'est pas allé trop loin en ICSI mais constate paradoxalement, avec Edwards [31], qu'en dépit de tous les risques, l'ICSI est de fait, comme en témoigne le suivi bien contrôlé des enfants d'ICSI, une procédure sûre.

\section{LE SUIVI CLINIQUE DES ENFANTS D'ICSI}

Une fréquence accrue d'aneuploides et de mosaïcismes a été rapportée [97], en diagnostic préimplantatoire, chez des embryons issus de prélèvements testiculaires dans des azoospermies non-obstructives en comparaison avec des embryons issus d'oligo-zoospermies. Aucune autre procédure de reproduction assistée n'a jamais été aussi bien suivie [112] dans sa progéniture que l'ICSI toutes indications comprises. Les suivis distincts des grossesses et des enfants issus d'ICSI avec prélèvement testiculaire dans des azoospermies non obstructives bien définies sont peu nombreux et souvent peu fiables [112]. II y a également fort peu d'informations sur les enfants nés de cellules germinales immatures [107].

Les anomalies les plus fréquemment signalées suite aux téchniques d'assistance à la reproduction et non spécifiques aux azoospermies non-obstructives, sont les malformations cardiaques (défauts de cloisonnement), uro-géni- tales (cryptorchidie en ICSI et hypospadias en FIV), et musculo-squelettiques (instabilité de la hanche). Après ICSI, les risques d'anomalies congénitales majeures varient entre $2,2 \%$ [57] à 4,1\% [123] et ceux d'anomalies mineures de $1,2 \%$ [57] à $6,3 \%$ [14]. En dépit de la controverse sur la définition même des anomalies majeures [11, 53] ces chiffres ne sont sensiblement guère différents des taux de malformations congénitales, généralement sousestimées, dans la population globale. Néanmoins, dans une étude récente portant sur 300 enfants d'ICSI et un groupe de contrôle, Hansen et coll. [43] trouvent un taux d'anomalies plus élevé en ICSI. Mais cette étude est controversée [112] sur le plan méthodologique en raison de séries discontinues d'enfants suivis, d'un groupe de contrôle mal apparié et de la connaissance anticipée par les examinateurs du type de conception.

Bowen et al. [15] dans une étude très critiquée [112] avait montré que le développement mental des enfants d'ICSI, surtout les garçons, était moins bon qu'après FIV ou conception naturelle. Là aussi le groupe de contrôle était mal apparié sur le plan ethnique et socioculturel. Ces résultats n'ont pas été corroborés dans une étude similaire plus grande de Bonduelle et al [12].

Il est intéressant de signaler une analyse multifactorielle non publiée de Ludwig et citée par Tournaye [112]. Dans cette étude, l'âge de la mère, la profession des parents, les maladies héréditaires familiales, l'usage du tabac ainsi que d'autres facteurs peuvent dans une certaine mesure augmenter le risque d'anomalies congénitales après ICSI mais la procédure d'ICSI elle-même n'est pas impliquée.

Ainsi, pour revenir au paradoxe de l'ICSI, il est permis avec Tournaye [112] de penser que l'injection intra-ovocytaire du spermatozoïde ne contourne pas toutes les étapes de la fécondation et du développement embryonnaire précoce. Tout se passe comme si l'ovocyte détenait encore des "postes de contrôle" permettant une sélection spermatique.

Puisqu'aucun risque spécifique tangible n'a pu être relevé après ICSI, à ce jour, peut-on arrêter la vigilance des suivis d'ICSI ? Certes non, elle doit se poursuivre ; Van Steirteghem et coll.[118], montrent, dans une étude portant sur 9781 enfants d'ICSI nés vivants, sans aucune augmentation des anomalies majeures en ICSI, par rapport aux enfants conçus naturellement, qu'il faut suivre 20.000 enfants d'ICSI et autant de contrôles appariés conçus naturellement, pour déceler une différence d'un demi pour cent du taux des anomalies, si l'on veut tenir compte des risques parentaux et de l'environnement socioculturel et économique. Bien que ce chiffre d'enfants ait déjà été dépassé, on ne dispose pas d'informations suffisantes sur chacun d'eux. Aussi, tant que ces statistiques n'atteindront pas la puissance statistique requise faut-il user de l'ICSI avec précaution et seulement s'il n'y a pas d'alternative [112].

\section{CONCLUSIONS}

Pour conclure, tous les candidats à l'ICSI doivent être rigoureusement explorés et informés surtout lorsqu'il 
s'agit d'azoospermie non-obstructive, à risque génétique plus élevé. II faut aussi les informer des limites de nos méthodes actuelles de prélèvements de spermatozoïdes testiculaires et de dépistage des risques génétiques et autres liés à la fécondation assistée dans toutes ses formes. Ils doivent aussi être informés de la possibilité d'une légère augmentation du taux d'anomalies chez leurs enfants, à cause sans doute de leurs propres risques héréditaires [112]. C'est aux patients eux-mêmes de jauger les avantages et les risques de ces traitements. Ainsi, si pour toutes sortes de raisons, le désir de la filiation biologique est fort, des couples peuvent en toute connaissance d'un risque héréditaire évident, opter [41] sciemment pour I'ICSI. Le diagnostic prénatal toujours recommandé en ICSI, ou même pré-implantatoire, s'impose particulièrement dans ces cas.

\section{REFERENCES}

1. ABOULGHAR M., MANSOUR R.T., SEROUR G.I. et al. : Fertilization and pregnancy rates after intracylasmic sperm injection using ejaculated semen and surgically retrieved sperm. Fertil. Steril., 1997, $68:$ 108-111.

2. ARIDOGAN I.A, BAYAZIT Y., YAMAN M., ERSOZ C., DORAN $S$. : Comparison of fine-needle aspiration and open biopsy of testis in sperm retrieval and histopathologic diagnosis. Andrologia, 2003, 35: 121-125.

3. AYTOZ A., CAMUS M.,TOURNAYE H., BONDUELLE M., VAN STEIRTEGHEM A., DEVROEY P. : Outcome of pregnancies after intracytoplasmic sperm injection and the effect of sperm origin and quality on this outcome. Fertil. Steril., 1998, 70 : 500-505.

4. BACHTELL N.E., CONAGHAN J., TUREK P.J. : The relative viability of human spermatozoa from the vas deferens, epididymis and testis before and after cryopreservation. Hum. Reprod., 1999, 14 : 3048-3051.

5. BALLESCA J.L., BALASCH J., CALAFELL J.M. et al. : Serum inhibin $B$ determination is predictive of successful testicular sperm extraction in men with non-obstructive azoospermia. Hum. Reprod., 2000, 15 : 1734-1738.

6. BARTOOV B., BERKOVITZ A., ELTES F., KOGOSOWSKI A., MENEZO Y., BARAK Y. : Real-time fine morphology of motile human sperm cells is associated with IVF-ICSI outcome. J. Androl., 2002, $23: 1-8$.

7. BENEZRA D. : In vitro fertilisation and retinoblastoma. Lancet, 2003, 361: 273-274.

8. BERGËRE M., SELVA J., VOLANTE M. et al. : Cytogenetic analysis of uncleaved oocytes after intracytoplasmic sperm injection. J. Assist. Reprod. Genet., 1995, 12 : 322-325.

9. BERNARDINI L., GIANAROLLI L., FORTINI D. et al. : Frequency of hyper-hypoploidy and diploidy in ejaculate, epididymal and testicular germ cells of infertile patients. Hum. Reprod., 2000, $15: 2165-2172$.

10. BICK D., FRANCO B., SHERINS R.J. et al. : Brief report : intragenic deletion of the KALIG-1 gene in Kalimann's syndrome. New England J. Med., 1992, 326 : 1752-1755.

11. BONDUELLE M., DEVROEY P., LIEBAERS I., VAN STEIRTEGHEM, A. : Major birth defects are overestimated. Brit. Med. J., 1997, 7118: 1265-1266.

12. BONDUELLE M., JORIS H., HOFMANS K., LIEBAERS I., VAN STEIRTEGHEM A.C. : Mental development of 201 child- ren at 2 years of age. Lancet, 1998, 351: 1535

13. BONDUELLE M., CAMUS M., DE VOS A, et al. : Seven years of intracytoplasmic sperm injection and follow-up of 1987 children. Hum. Reprod., 1999, $14:$ 243-264.

14. BONDUELLE M., LIEBAERS I., DEKETELAERE V. et al. : Neonatal data on a cohort of 2889 infants born after ICSI (1991-1999) and of 2995 infants born after IVF (1983-1999). Hum. Reprod., 2000, 17 : 671-694.

15. BOWEN J.R., GIBSON F.L., LESLIE G.I., SAUNDERS D.M. : Medical and developmental outcome at 1 year for children conceived by intracytoplasmic sperm injection. Lancet, 1998, 351: 1529-1534.

16. BRANDELL R.A., MIELNIK A., LIOTTA D. et al. : AZFb deletions predict the absence of spermatozoa with testicular sperm extraction : preliminary report of a prognostic genetic test. Hum. Reprod., 1998, $13: 2812-2815$.

17. BRENNER C.A., BARRITT J.A., WILLADSEN,S., COHEN J. : Mitochondrial DNA heteroplasmy after human ooplasmic transplantation. Fertil. Steril., 2000, $74: 573-578$.

18. BROSSFIELD J.E., CHAN P.J., PATTON W.C., KING A. : Tenacity of exogenous human papillomavirus DNA in sperm washing. J. Assist. Reprod. Genet., 1999, 16 : 325-328.

19. BRUGO-OLMEDO S., DE VINCENTIIS S., CALAMERA J.C., URRUTIA F., NODAR, F., ACOSTA A. : Serum inhibin B may be a reliable marker of the presence of testicular spermatozoa in patients with nonobstructive azoospermia. Fertil. Steril., 2001, 76: 1124-1129.

20. CALOGERO A.E., BURRELLO N., DE PALMA A., BARONE N., D'AGATA R., VICARI E. : Sperm aneuploidy in infertile men. Review. Reprod. BioMed. Online, 2003, 6 : 310-317.

21. CHAN A.W., LUETJENS C.M., DOMINKO T. : Transgen ICSI reviewed: foreign DNA transmission by intracytoplasmic sperm injection in rhesus monkey. Mol. Reprod. Develop., 2000, 56 : 325-328.

22. CRAM D.S., SONG B., MCLACHLAN R.I., TROUNSON A.O. : CAG trinucleotide repeats in the androgen receptor gene of infertile men exhibit stable inheritance in female offspring conceived after ICSI. Mol. Hum. Reprod., 2000, 6 : 861-866.

23. CUMMINS J.M. : Fertilization and elimination of the paternal mitochondrial genome. Review. Hum. Reprod., 2000, 15 : (Suppl 2) 92-101.

24. DEVROEY P., LIU J., NAGY Z., TOURNAYE H., SILBER S.J., VAN STEIRTEGHEM A.C. : Normal fertilization of human oocytes after testicular sperm extraction and intracytoplasmic sperm injection. Fertil. Steril., 1994, 62 : 639-641.

25. DEVROEY P., LIU J., NAGY Z. : Pregnancies after testicular sperm extraction and intracytoplasmic sperm injection in nonobstructive azoospermia. Hum. Reprod., 1995, 10 :14571460.

26. DE CROO I., VAN DER ELST J., EVERAERT K., DE SUTTER P., DHONT M. : Fertilization, pregnancy and embryo implantation rates after ICSI in cases of obstructive and non-obstructive azoospermia. Hum. Reprod., 2000, 15 : 1383-1388.

27. DHONT M., DE SUTTER P., RUYSSINCK G., MARTENS G., BEKAERT A. Perinatal outcome of pregnancies after assisted reproduction:a case-control study. Am. J. Obst. Gynecol., 1999, $181: 688-695$.

28. DOWSING A.T., YONG E.L., CLARK M., MCLACHLAN R., DE KRETSER D.M., TROUNSON A.O. : Linkage between male infertility and trinucleotide repeat expansion in the androgenreceptor gene. Lancet, 1999, 354:640-643.

29. DOYLE P., BERAL V., MACONOCHIE N. : Preterm delivery, 
low birthweight and small-for-gestational-age in liveborn singleton babies resulting from in vitro fertilization. Hum. Reprod., 1992, $7:$ :425-428.

30. EDIRISINGHE W.R., MURCH A., JUNK S., YOVICH J.L. : Cytogenetic abnormalities of unfertilized oocytes generated from in vitro fertilization and intracytoplasmic sperm injection : a double-blind study. Hum. Reprod., 1997, 12 : 2784-2791.

31. EDWARDS R.G. : Widening perspectives of intracytoplasmic sperm injection. Nature Med., 1999, $5: 377-378$.

32. EDWARDS R.G., LUDWIG M. : Are major defects in children conceived in vitro due to innate problems in patients or to induced genetic damage ? < www.rbmonline.com/Article/927 $>$ Reprod. Biomed. Online, 2003, 7 : (sous presse).

33. EGOZCUE J., BLANCO J., VIDAL F. : Chromosome studies in human sperm nuclei using fluorescence in-situ hybridization (FISH). Hum. Reprod. Update, 1997, 3 : 441-452.

34. EYTAN O., HAR-TOOV J., FAIT G. et al. : Vascularity index distribution within the testis : a technique for guiding testicular sperm extraction. Ultrasound Med. Biol., 2001, 27 : 11711176.

35. FRIEDLER S., RAZIEL A., STRASSBURGER D., SOFFER Y., KOMAROVSKY D., RON-EL R. : Testicular sperm retrieval by percutaneous fine needle sperm aspiration compared with testicular sperm extraction by open biopsy in men with non-obstructive azoospermia. Hum. Reprod., 1997, 12 : 1488-1493.

36. FRIEDLER S., RAZIEL A., SCHACHTER M., STRASSBURGER D., BERN O., RON-EL R. : Outcome of first and repeated testicular sperm extraction and ICSI in patients with nonobstructive azoospermia. Hum. Reprod., 2002, 17 : 23562361.

37. FRIEDLER S., RAZIEL A., STRASSBURGER D., SCHACHTER M., SOFFER Y., RON-EL R. : Factors influencing the outcome of ICSI in patients with obstructive and non-obstructive azoospermia : a comparative study. Hum. Reprod., 2002, 17 : 114-121.

38. GARDNER R.L. : Scrambled or bisected mouse eggs and the basis of patterning in mammals. Review. Bioessays, 1999, 21: $271-274$

39. GHAZZAWI I.M., SARAF M.G., TAHER M.R., KHALIFA F.A. : Comparison of fertilizing capability of spermatozoa from ejaculates, epididymal aspirates and testicular biopsies using intracylasmic sperm injection. Hum. Reprod., 1998, 13 : 348-352.

40. GIL-SALOM M., ROMERO J., MINGUEZ Y., MOLERO M.D., REMOHI J., PELLICER A. : Testicular sperm extraction and intracylasmic sperm injection : a chance of fertility in non-obstructive azoospermia. J. Urol., 1998, $160:$ 2063-2067.

41. GILTAY J.C., KASTROP P.M., TUERLINGS J.H. et al. : Subfertile men with constitutive chromosome abnormalities do not necessarily refrain from intracytoplasmic sperm injection treatment: a follow-up study on 75 Dutch patients. Hum. Reprod., 1999, 14, 318-320.

42. GOSDEN R., TRASLER J., LUCIFERO D., FADDY M. : Rare congenital disorders, imprinted genes, and assisted reproductive technology, Review. Lancet, 2003, 361 : 1975-1977.

43. HANSEN M., KURINCZUK J.J., BOWER C., WEBB S. : The risk of major birth defects after intracytoplasmic sperm injection and in vitro fertilization. New Engl. J. Med., 2002, 346 : 725-730.

44. HEWITSON L., TAKAHASHI D., DOMINKO T., SIMERLY C., SCHATTEN G. : Fertilization and embryo development to blastocysts by intracytoplasmic sperm injection in the rhesus monkey. Hum. Reprod., 1998, 13 : 2786-2790.
45. HEWITSON L., DOMINKO T., TAKAHASHI D. et al. : Unique checkpoints during the first cell cycle of fertilization after intracytoplasmic sperm injection in rhesus monkeys. Nature Med., 1999, $5: 431-433$.

46. KAHRAMAN S., OZGUR S., ALATAS C. et al. : High implantation and pregnancy rates with testicular sperm extraction and intracylasmic sperm injection in obstructive and non-obstructive azoospermia. Hum. Reprod., 1996, 11 : 673-676.

47. KENT-FIRST M.G., KOL S., MUALLEM A. et al. : The incidence and possible relevance of $Y$-linked microdeletions in babies born after intracytoplasmic sperm injection and their infertile fathers. Mol. Hum. Reprod., 1996, 2 : 943-950.

48. KIMURA Y., YANAGIMACHI R. : Development of normal mice from oocytes injected with secondary spermatocyte nuclei. Biol. Reprod., 1995, $53:$ 855-862.

49. KLEIMAN S.E., MAYMON B.B., YOGEV L., PAZ G., YAVETZ $H$. : The prognostic role of the extent of $Y$ microdeletion on spermatogenesis and maturity of Sertoli cells. Review. Hum. Reprod., 2001, 16 SD: 399-402.

50. KOVALEVSKY G., RINAUDO P., COUTIFARIS C. : Do assisted reproductive technologies cause adverse fetal outcomes? Fertil. Steril., 2003, $79:$ 1270-1272.

51. KREMER J.A.,TUERLINGS J.H., BORM G. et al. : Does intracytoplasmic sperm injection lead to a rise in the frequency of microdeletions in the AZFc region of the $Y$ chromosome in future generations ? Hum. Reprod., 1998, 13 : 2808-2811.

52. KUPKER W., SCHULZE W., DIEDRICH K. : Ultrastructure of gametes and intracytoplasmic sperm injection : the significance of sperm morphology. Mol. Hum. Reprod., 1998, 13 (Suppl. 1) : $99-106$

53. KURINCZUK J.J., BOWER C. : Birth defects in infants conceived by intracytoplasmic sperm injection-an alternative interpretation. Brit. Med. J., 1997, 315 : 1260-1265.

54. LACEY R.W., DEALLER S.F. : Vertical transfer of prion disease. Hum. Reprod., 1994, 9 : 1792-1800.

55. LANZENDORF S.E., MALONEY M.K., VEECK L.L., SLUSSER J., HODGEN G.D., ROSENWAKS Z. : A preclinical evaluation of pronuclear formation by microinjection of human spermatozoa into human oocytes. Fertil. Steril., 1988, 49 : 835-842.

56. LEWIN L.M., LEVEK-MOTOLA N., GOLAN R. et al. : Flow cytometric ploidy analysis of semen cells as a non-invasive diagnostic method for assessing spermatogenesis in azoospermic patients. In: Proceedings Vol. 15th Congress EuroMedLab, Barcelona 2003. Bologna, Monduzzi Editore Spa, 2003.

57. LOFT A., PETERSEN K., ERB. K. et al. : A Danish national cohort of 730 infants born after intracytoplasmic sperm injection (ICSI)1994-1997. Hum. Reprod., 1999, 14 : 2143-2148.

58. LUDWIG M., SCHROEDER A.K., DIEDRICH K. : Impact of intracytoplasmic sperm injection on the activation and fertilization process of oocytes. Reprod. BioMed. Online, 2001, 3 : 230-240.

59. LUETJENS C.M., PAYNE C., SCHATTEN G. : Non-random chromosome positioning in human sperm and sex chromosome anomalies following intracytoplasmic sperm injection. Lancet, 1999, $353: 1240$.

60. MANSOUR R.T., KAMAL A., FAHMY I., TAWAB N., SEROUR G.I., ABOULGHAR M.A. : Intracylasmic sperm injection in obstructive and non-obstructive azoospermia. Hum. Reprod., 1997, $12:$ 1974-1979.

61. MAYMON B.B., YOGEV L., PAZ G. et al. : Sertoli cell matura- 
tion in men with azoospermia of different etiologies. Fertil. Steril., 2002, 77 : 904-909.

62. MCINNES B., RADEMAKER A., GREENE C.A., KO E., BARCLAY L., MARTIN R.H. : Abnormalities for chromosomes 13 and 21 detected in spermatozoa from infertile men. Hum. Reprod., 1998, $13: 2787-2790$

63. MERCAN R., URMAN B., ALATAS C, et al. : Outcome of testicular sperm retrieval procedures in non-obstructive azoospermia : precutaneous aspiration versus open biopsy. Hum. Reprod., 2000, $15: 1548-1551$.

64. MESCHEDE D., LEMCKE B., BEHRE H.M., DE GEYTER C., NIESCHLAG, E., HORST J. : Clustering of male infertility in the families of couples treated with intracytoplasmic sperm injection. Hum. Reprod., 2000, 15 : 1604-1608.

65. MESCHEDE D., LEMCKE B., BEHRE H.M., DE GEYTER C.D., NIESCHLAG, E. HORST J. : Non-reproductive heritable disorders in infertile couples and their first degree relatives. Hum. Reprod., 2000, $15: 1609-1612$.

66. MIHARU N., BEST R.G., YOUNG S.R. : Numerical chromosome abnormalities in spermatozoa of fertile and infertile men detected by fluorescence in situ hybridization. Hum. Genetics, 1994, 93 : 502-506.

67. MILLER D., BRIGGS D., SNOWDEN H. et al. : A complex population of RNAs exists in human ejaculate spermatozoa : implications for understanding molecular aspects of spermiogenesis. Gene, 1999, 237 : 385-392.

68. MOLL A.C., IMHOF S.M., CRUYSBERG J.R., SCHOUTENVAN MEETEREN A.Y., BOERS M., VAN LEEUWEN F.E. Incidence of retinoblastoma in children born after in vitro fertilisation. Lancet, 2003, $361: 309-310$.

69. MULHALL J.P., BURGESS C.M., CUNNINGHAM D., CARSON R., HARRIS. D., OATES R.D. : Presence of mature sperm in testicular parenchyma of men with non-obstructive azoospermia : prevalence and predictive factors. Urology, 1997, $49: 91-96$.

70. NAGY Z., LIU J., JORIS H., BOCKEN G. et al. : The result of intracytoplasmic sperm injection is not related to any of the three basic sperm parameters. Hum. Reprod., 1995, 10 : 1123-1129.

71. PAGE D.C., SILBER S., BROWN L.G. : Men with infertility caused by AZFc deletion can produce sons by intracytoplasmic sperm injection, but are likely to transmit the deletion and infertility. Hum. Reprod., 1999, 14 :1722-1726.

72. PALERMO G.P., JORIS H., DEVROEY P., VAN STEIRTEGHEM A.C. : Pregnancies after intracytoplasmic injection of single spermatozoon into an oocyte. Lancet, 1992, $340: 826-$ 835 .

73. PALERMO G.D., COLOMBERO L.T., ROSENWAKS Z. : The human sperm centrosome is responsible for normal syngamy and early embryonic development. Rev. Reprod., 1997, 2 : 1927.

74. PALERMO G.D., SCHLEGEL P.N., HARIPRASHAD J.J. et al.: Fertilization and pregnancy outcome with intracylasmic sperm injection for azoospermic men. Hum. Reprod., 1999, 14 : 741748

75. PALERMO G.D., TAKEUCHI T., NERI Q.V., KATAGIRI Y,. VEECK L.L., ROSENWAKS Z : Application of intracytoplasmic sperm injection in assisted reproductive technologies. Reprod. BioMed., 2003, 6 : 456-463.

76. PANG M.G., HOEGERMAN S.F., CUTICCHIA A.J. et al. : Detection of aneuploidy for chromosomes $4,6,7,8,9,10,11$, $12,13,17,18,21, X$ and $Y$ by fluorescence in situ hybridiza- tion in spermatozoa from nine patients with oligoastheno-teratozoospermia undergoing intracytoplasmic sperm injection. Hum. Reprod., 1999, 14 : 1266-1273.

77. PAZ G., GAMZU R., YAVETZ H. : Diagnosis of nonobstructive azoospermia : the laboratory perspective. Review. J. Androl., 2003, $24: 167-169$

78. PERRY A.C.F., WAKAYAMA T., KISHIKAWA H., KASAI M., TOYODA Y., YANAGIMACHI R. : Mammalian transgenesis by intracytoplasmic sperm injection. Science, 1999, $284: 1180-$ 1183.

79. PETERSEN K., HORNNES P.J., ELLINGSEN S. et al. : Perinatal outcome after in vitro fertilization. Acta Obstet. Gynecol. Scandinav., 1995, $74: 129-131$.

80. PIOTROWSKA K., ZERNICKA-GOETZ M. : Role for sperm in spatial patterning of the early mouse embryo. Nature, 2001, $409: 517-521$.

81. PLACHOT $M$. : Les risques génétiques spécifiques de l'ICSI. Contracept. Fertil. Sexual., 1996, 24 : 577-580.

82. RON-EL R., STRAUSS S., FRIEDLER S., STRASSBURGER D., KOMAROVSKY D., RAZIEL A. : Serial sonography and colour flow Doppler imaging following testicular and epididymal sperm extraction. Hum. Reprod., 1998, 13 : 3390-3393.

83. RON-EL R., STRASSBURGER D., FRIEDLER S., KOMAROVSKY D., BERN O., RAZIEL A. : Repetitive ejaculation before intracytoplasmic sperm injection in patients with absolute immotile spermatozoa. Hum. Reprod., 1998, $13: 630$ 633.

84. SANTHANANTHAN A.H., KOLA I., OSBORNE J. et al. : Centrioles in the beginning of human development. Review. Proc. Natl. Acad. Sci. USA, 1991, $88:$ 4806-4810.

85. SCHATTEN G. : The centrosome and its mode of inheritance: the reduction of the centrosome during gametogenesis and its restoration during fertilisation. Dev. Biol., 1994, $165: 299-325$.

86. SCHATTEN G., HEWITSON L., SIMERLY C., SUTOVSKY P., HUSZAR G. : Cell and molecular biological challenges of ICSI: ART before science ? J Law Med. Ethics, 1998, 26 : 29-37.

87. SCHATTEN G.P. : Safeguarding ART. Nature Cell Biol., Fertility Suppl., 2002, 4(S1) : S19-S22.

88. SCHIEVE L.A., MEIKLE S.F., FERRE C., PETERSON H.B., JENG G., WILCOX L.S. : Low and very low birth weight in infants conceived with assisted reproductive technology low and very low birth weight in infants conceived with use of assisted reproductive technology. New Engl. J. Med., 2002, $346: 731-737$

89. SCHLEGEL P.N. : Testicular sperm extraction: microdissection improves sperm yield with minimal tissue excision. Hum. Reprod., 1999, $14:$ 131-135.

90. SCHOYSMAN R., VANDERZWALMEN P., NIJS M. et al. : A pregnancy obtained with human testicular spermatozoa in an in vitro fertilization program. J. Androl. 1994, 15(Suppl) : 10S$13 \mathrm{~S}$.

91. SHUFARO Y., PRUS D., LAUFER N., SIMON A. : Impact of repeated testicular fine needle aspirations (TEFNA) and testicular sperm extraction (TESE) on the microscopic morphology of the testis : an animal model Hum. Reprod., 2002, 17 : 17951799.

92. SIFFROI J.P., LE BOURHIS C., KRAUSZ C. et al. : Sex chromosome mosaicism in males carrying $Y$ chromosome long arm deletions. Hum. Reprod., 2000, 15 : 2559-2562.

93. SIFFROI J.P., CHANTOT-BASTARAUD S., RAVEL C. : Origines géniques et chromosomiques des anomalies de la spermatogenèse : aspects cliniques et rapports avec les modèles 
animaux. Gynécol. Obstét. Fertil., 2003, 31 : 504-515.

94. SILBER S.J., VAN STEIRTEGHEM A.C., LIU J., NAGY Z., TOURNAYE H., DEVROEY P. : High fertilization and pregnancy rate after intracytoplasmic sperm injection with spermatozoa obtained from testicle biopsy. Hum. Reprod., 1995, 10 : 148-152.

95. SILBER S.J., NAGY Z., DEVROEY P., CAMUS M., VAN STEIRTEGHEM A.C. : The effect of female age and ovarian reserve on pregnancy rate in male infertility : treatment of azoospermia with sperm retrieval and intracylasmic sperm injection. Hum. Reprod., 1997, 12 : 2693-2700.

96. SILBER S.J., ALAGAPPAN R., BROWN L.G., PAGE D.C. : Y chromosome deletions in azoospermic and severely oligozoospermic men undergoing intracytoplasmic sperm injection after testicular sperm extraction. Hum. Reprod., 1998, 13 : 3332-3337.

97. SILBER S.J., REPPING S. : Transmission of male infertility to future generations : lessons from the $Y$ chromosome. Hum. Reprod. Update, 2002, $8: 217-229$.

98. SILVA C.P., KOMMINENI K., OLDENBOURG R., KEEFE D.L.: The first polar body does not predict accurately the location of the metaphase II meiotic spindle in mammalian oocytes. Fertil. Steril., 1999, $71:$ 719-721.

99. SIMERLY C., WU G.J., ZORAN S. : The paternal inheritance of the centrosome, the cell's microtubule-organizing center, in humans, and the implications for infertility. Nature Med., 1995, $1: 47-52$.

100.SIMONI M., BAKKER E., EURLINGS M.C.M. et al. : Laboratory guidelines for molecular diagnosis of Y-chromosomal microdeletions. Int. J. Androl., 1999, 22 : 292-299.

101.SIMONI M. : Molecular diagnosis of $Y$ chromosome microdeletions in Europe : state-of-the-art and quality control. Hum. Reprod., 2001, 16 : 402-409.

102.ST JOHN J.C. : Ooplasm donation in humans. The need to investigate the transmission of mitochondrial DNA following cytoplasmic transfer. Hum. Reprod., 2002, 17 : 1954-1958.

103.STRASSBURGER D., REICHART M., KAUFMAN S. et al. : Morphology and fluoresence in situ hybridization on the same sperm cell controlled by computerized cell scanning system. ESHRE 2003 Abst O-231. Hum. Reprod., 2003, 18 (Suppl 1) : 79-80.

104.TALLO C.P., VOHR B., OH W., RUBIN L.P., SEIFER D.B., HANING R.V. JR. : Maternal and neonatal morbidity associated with in vitro fertilization. J. Pediatr., 1995, $127: 794-800$.

105.TARLATZIS B., BILI H. : Survey on intracylasmic sperm injection : report from the ESHRE ICSI Task Force. Hum. Reprod., 1998, 13 (Suppl.1) : 165-177.

106.TASDEMIR I., TASDEMIR M., TAVUKCUOGLU S., KAHRAMAN S., BIBEROGLU K. : Effect of abnormal sperm head morphology on the outcome of intracytoplasmic sperm injection in humans. Hum. Reprod., 1997, 12 : 1214-1217.

107.TESARIK J., MENDOZA C. : Using the male gamete for assisted reproduction : past, present and future. J. Androl., 2003, 24 : 317-328.

108. TOURNAYE H., LIU J., NAGY P.Z. et al. : Correlation between testicular histology and outcome after intracylasmic sperm injection using testicular spermatozoa. Hum. Reprod., 1996, 11:127-132.

109.TOURNAYE H., LIU J., NAGY Z., VERHEYEN G., VAN STEIRTEGHEM A.C., DEVROEY P. : The use of testicular sperm for intracytoplasmic sperm injection in patients with necro-zoospermia. Fertil. Steril., 1996, 66 : 331-334.
110. TOURNAYE H., VERHEYEN G., NAGY P. et al. : Are there any predictive factors for successful testicular sperm recovery in azoospermic patients ? Hum. Reprod., 1997, $12: 80-86$.

111. TOURNAYE H., LISSENS W., LIEBAERS I. et al. : Heritability of sterility: clinical implications. In : Genetics of Human Male Fertility. Barratt C., De Jonge C., Mortimer D., Parinaud D. eds. Paris, Editions EDK, 1997 : 123-144.

112. TOURNAYE H. : ICSI, too far ? Int. J. Androl., 2003, 26 : 6369.

113. TSUJIMURA A., MATSUMIYA K., MIYAGAWA Y. et al. : Conventional multiple or microdissection testicular sperm extraction : a comparative study. Hum. Reprod., 2002 : 17 : 2924-2929.

114. TUT T.G., GHADESSY F.J., TRIFIRO M.A., PINSKY L., YONG E.L. : Long polyglutamine tracts in the androgen receptor are associated with reduced trans-activation, impaired sperm production, and male infertility. J. Clin. Endocrinol. Metab., 1997, 82 : 3777-3782.

115. UBALDI F., NAGY Z.P., RIENZI L. et al. : Reproductive capacity of spermatozoa from men with testicular failure. Hum. Reprod., 1999, 14 : 2796-2780.

116. VAN GOLDE R., BOADA M., VEIGA A., EVERS J., GERAEDTS J., BARRI P. : A retrospective follow-up study on intracyto-plasmic sperm injection. J. Assist. Reprod. Genet., $1999,16: 227-232$.

117. VAN BLERKOM J. : Sperm centrosome dysfunction: a possible new class of male factor infertility in the human. Mol. Hum. Reprod., 1996, 2 : 349-354.

118. VAN STEIRTEGHEM A., BONDUELLE M., DEVROEY P., LIEBAERS I. : Follow-up of children born after ICSI. Hum. Reprod. Update, 2002, $8: 111-116$.

119. VERNAEVE V., TOURNAYE H., SCHIETTECATTE J., VERHEYEN G., VAN STEIRTEGHEM A., DEVROEY P. : Serum inhibin $B$ cannot predict testicular sperm retrieval in patients with non-obstructive azoospermia. Hum. Reprod., 2002, 17 : 971-976.

120.VERNAEVE V., TOURNAYE H., OSMANAGAOGLU K., VERHEYEN G., VAN STEIRTEGHEM A., DEVROEY P. : Intracytoplasmic sperm injection with testicular spermatozoa is less successful in men with nonobstructive azoospermia than in men with obstructive azoospermia. Fertil. Steril., 2003, 79 : 529-533.

121. VICARI E., GRAZIOSO C., BURRELLO N., CANNIZZARO M., D'AGATA R., CALOGERO A.E. : Epididymal and testicular sperm retrieval in azoospermic patients and the outcome of intracylasmic sperm injection in relation to the etiology of azoospermia. Fertil. Steril., 2001, $75: 215-216$.

122.VON ECKARDSTEIN S., SIMONI M., BERGMANN M. et al. : Serum inhibin $B$ in combination with serum follicle-stimulating hormone $(\mathrm{FSH})$ is a more sensitive marker than serum $\mathrm{FSH}$ alone for impaired spermatogenesis in men, but cannot predict the presence of sperm in testicular tissue samples. J. Clin. Endocrinol. Metab., 1999, 84 : 2496-2501.

123.WENNERHOLM U.B., BERGH C., HAMBERGER L. : Incidence of congenital malformations in children born after ICSI. Hum. Reprod., 2000, $15: 944-948$.

124.WESTLANDER G., HAMBERGER L., HANSON C. : Diagnostic epididymal and testicular sperm recovery and genetic aspects in azoospermic men. Fertil. Steril., 1999, 14 : 118122.

125.WESTLANDER G., EKERHOVD E., GRANBERG S. et al. : Serial ultrasonography, hormonal profile and antisperm anti- 
body response after testicular sperm aspiration. Hum. Reprod., 2001, 16 : 2621-2627.

126.WINSTON R., HARDY K. : Are we ignoring potential dangers of in vitro fertilization and related treatments ? Nature Cell Biol., 2002, 4(S1) : 14-18.

127. WISANTO A., BONDUELLE M., CAMUS M. : Obstetric outcome of 904 pregnancies after intracytoplasmic sperm injection. Hum. Reprod., 1996, 11 : 121-129.

128. YANAGIMACHI R. : Intracytoplasmic sperm injection experiments using the mouse as a model. Hum. Reprod., 1998,13 (Suppl.1) : 87-98.

129. YAVETZ H., YOGEV L., KLEIMAN S. et al. : Morphology of testicular spermatozoa obtained by testicular sperm extraction in obstructive and nonobstructive azoospermic men and its relation to fertilization success in the in vitro fertilizationintracytoplasmic sperm injection system. J. Androl., 2001, 22 : 376-381.

130. YOGEV L., GAMZU R., PAZ G. : Rate of homologous chromosome bivalents in spermatocytes may predict completion of spermatogenesis in azoospermic men. Hum. Genet., 2002, $110: 30-35$.

131. YOSHIDAA., MIURA K., SHIRAI M. : Chromosome abnormalities and male infertility. Assisted Reprod. Rev., 1996, 6 : 9399.

132.ZERNICKA-GOETZ M. : Patterning of the embryo : the first spatial decisions in the life of a mouse. Development, 2002, $129: 815-829$.

Manuscrit reçu : août 2003 ; accepté : décembre 2003

\section{ABSTRACT}

Nonobstructive azoospermia : predictive factors of testicular sperm retrieval and risks of assisted fertilization

\section{Yigal SOFFER}

Surgical sperm retrieval has revolutionized the treatment of azoospermia. Intracytoplasmic sperm injection (ICSI) allows naturally infertile men to have children by allowing defective sperm cells to fertilize oocytes. These techniques, applied without any preliminary animal experimentation, raised an enormous enthusiasm and are performed on a large-scale. To increase the efficiency of these treatments, the clinicians are now trying, without success, to identify factors predictive of success allowing better patient selection and counselling of couples dependent on these techniques in order to avoid useless and harmful interventions. Animal research, conducted after introduction of these techniques, has raised serious doubts about the safety of ICSI and the legitimacy of using defective spermatozoa from genetically high-risk patients. Some studies have also emphasized the unusual frequency of obstetric and neonatal problems as well as rare diseases and malignancies in ICSI-born children and ART-born children. However, these disturbing findings are not specifically related to the ICSI procedure, as demonstrated by well-conducted large-scale follow-up studies in ICSI-born children. This paradox raises a lively debate. ICSI-children follow-up studies should continue until sound data taking into account the genetic and all other parental background are obtained. In conclusion, non obstructive azoospermic patients should be informed of the limits of sperm retrieval and genetic screening tools as well as all risks common to ICSI and ART.

Key-Words: male Infertility, nonobstructive azoospermia, testicular sperm retrieval, ICSI, ART, factors of success, genetic risks 